\title{
Endoscopic ear surgery in the ear camp setting; forward thinking or folly?
}

\author{
M P A CLARK
}

ENT Department, Gloucestershire Royal Hospitals NHS Trust, UK, and British Nepal Otology Service ('BRINOS') Ear Care Centre, Nepalgunj, Nepal

\begin{abstract}
Background: Endoscopic ear surgery is becoming an accepted technique in otological surgery, in the management of chronic otitis media.

Methods: The technique was introduced to the humanitarian care setting of an ear camp in Nepal to consider the appropriateness of the technique in this type of clinical setting.

Results: Fifteen cases of myringoplasty were successfully completed.

Conclusion: The apparent advantages of the endoscopic approach over the traditional microscopic one were the ease of transporting the equipment and the optimal view obtained of the tympanic membrane. The ability for pathology and operative technique to be observed equally well by the surgeon and local staff was helpful for teaching, and enables a move towards self-sufficiency of care. Easy image capture also has potential for remote telemedicine applications.
\end{abstract}

Key words: Tympanic Membrane Perforation; Endoscopes; Global Health; Education

\section{Introduction}

The endoscope has now found a definitive, accepted place in ear surgery. ${ }^{1-3}$ The virtues of being able to view and access the challenging recesses of the middle ear are well documented, where the endoscope serves well as an adjunct to the standard microscopic approach. Cholesteatoma may be tackled entirely endoscopically, particularly where disease is more limited, such that an atticotomy-type approach will achieve disease clearance. Myringoplasty techniques are also well suited to the endoscope, obviating the need for a microscope at all. Anterior perforations are easily viewed, tortuous ear canals are more easily negotiated and a post-auricular scar can be avoided. This may allow for an increase in the day-case rate and a reduction in post-operative pain. In the clinic setting, the endoscope equally has its value; it provides a beautifully bright, wide-angled view of the tympanic membrane that can be observed as a whole, rather than the quadrant approach necessary with a standard auriscope.

Perhaps the greatest advantage of the endoscopic approach comes with the attachment of a camera, where the image, either in the out-patient department or the operating theatre, is viewed on a television screen. Whilst the camera is not the preserve of the endoscope, when combined, the image seen by the surgeon is shared equally with all others present - be it the patient in the clinic or the operating theatre staff and trainees. This is in contrast to operating with a microscope.

The reality is that many of the microscopes available for use in the ear camp setting lack the side arm for camera attachment. When available, the view obtained during microscopic surgery is not the binocular view seen by the surgeon, so those observing are not truly seeing what the surgeon is seeing. The broadcast image is often off-centre, poorly focused or mal-rotated, and many surgeons lose tolerance to continually adjust it whilst trying to concentrate on the job in hand. The endoscope with camera setup removes these issues.

\section{Materials and methods}

Thanks to a very generous donation from Storz, a new set of endoscopic ear surgery equipment was taken to the 59th ear camp at the new British Nepal Otology Service ('BRINOS') Ear Care Centre in Nepalgunj. It is important to consider the appropriateness of using new technology and techniques when setting up ear camps in the developing world. The essentials of the equipment were: a Telepack (TP $100-$ Tele Pack X LED) with keyboard; a Telecam one-chip camera head; $3 \mathrm{~mm}$ diameter, $14 \mathrm{~cm}$ length Hopkins telescopes; and micro-instruments.

The entire set of equipment was transported in a small hard suitcase, making it easily portable yet protected (Figure 1). This proved to be the first advantage in the ear camp setting. The transportation of operating microscopes is a logistical challenge, as they are large, with delicate optics and balancing systems. In contrast, the size of the packed endoscopic ear surgery kit would even comply with some airline handluggage requirements and weighed less than $14 \mathrm{~kg}$.

\section{Results}

At the camp, we performed 15 myringoplasties using an endoscopic technique (our number was limited by some technical teething problems). The patients were managed in 
(a)

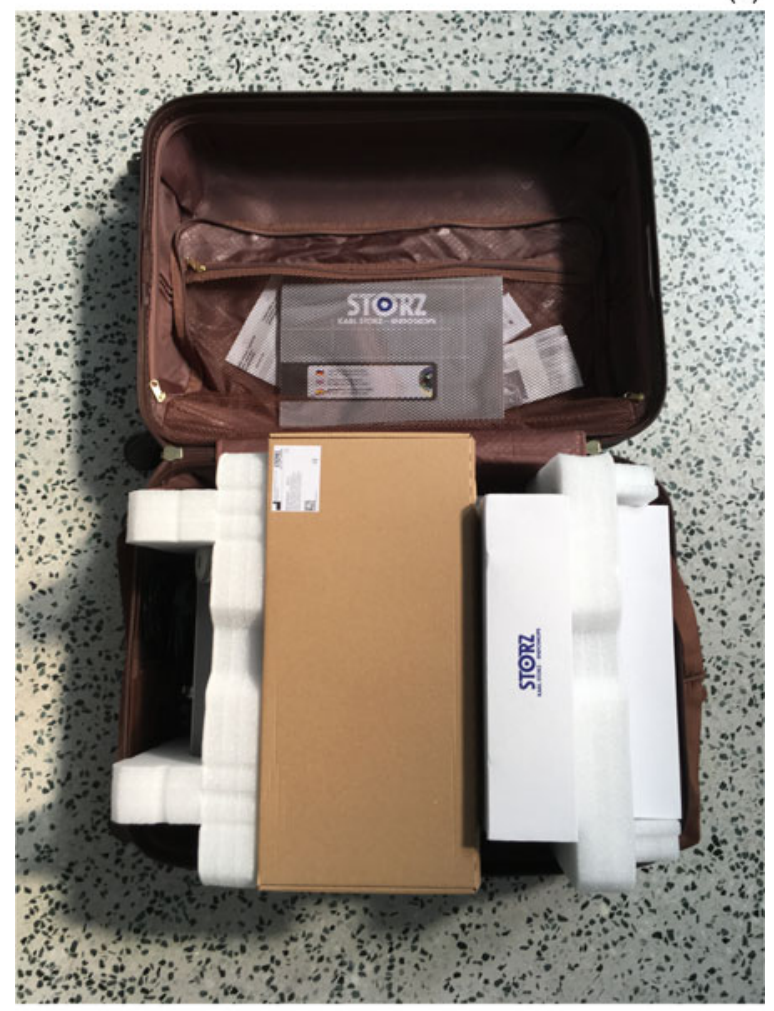

(b)

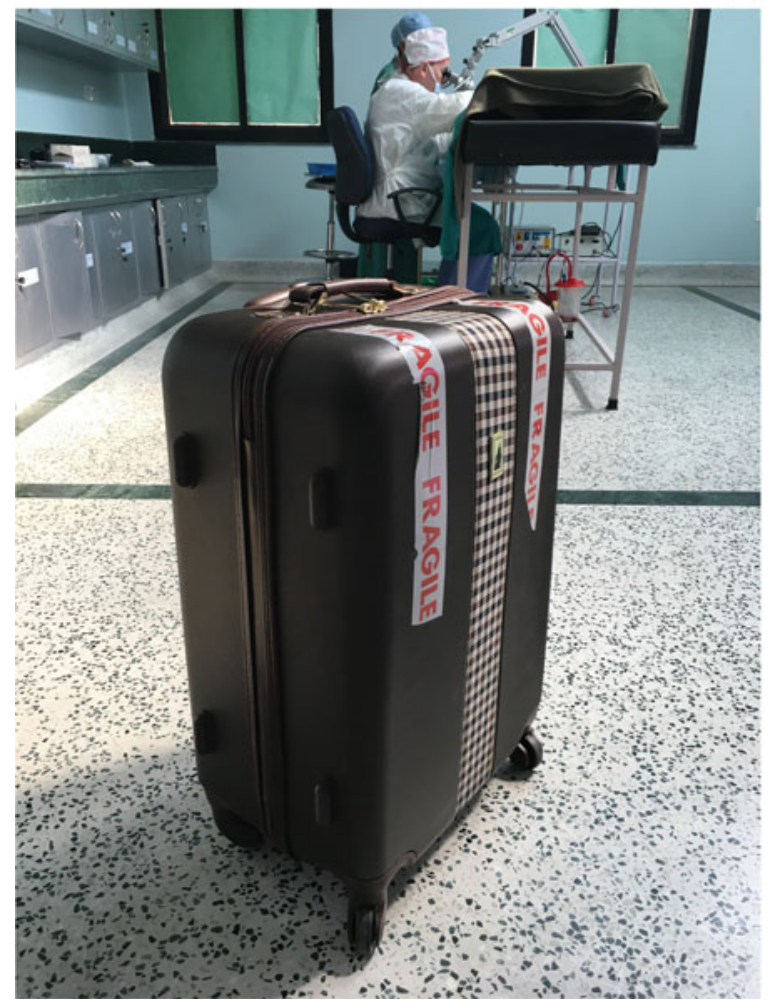

FIG. 1

(a \& b) Entire endoscopic ear surgery equipment packed for transport or storage.

the same way as for microscopic repair, except for the use of the endoscope. The procedures were performed under local anaesthesia, administered in the same manner for both techniques. Case selection was conducted at random from the day's full list, rather than based on specific patient

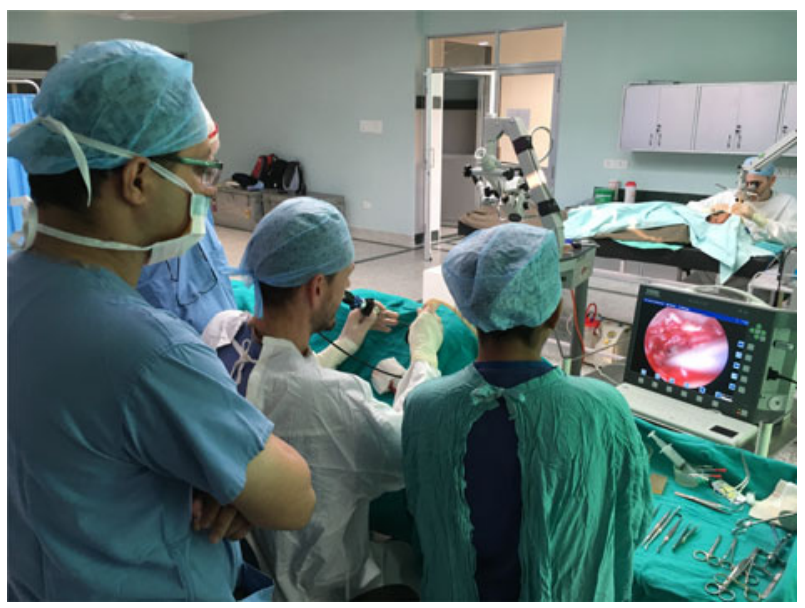

FIG. 2

Endoscopic ear surgery in progress.

demographics or tympanic membrane perforation size or sites. Whilst operation timing was not specifically measured for these cases, no alterations were made to the day's surgery schedule sizes to accommodate the new technique.

Overall, several advantages were observed. An optimal operating view was obtained - the microscopes otherwise at our disposal are old and always prove somewhat of a challenge to use (the sticker on one stating Carl Zeiss, West Germany, is a giveaway to its age). Anterior perforations were easily viewed, removing the need for a post-auricular approach. All cases were completed using a transcanal technique. Some cases that were started with the microscope were converted to the endoscopic approach for this reason.

Of greatest value, were the comments received from the Nepalese scrub nurses. It was clear that, for the first time, they could see and understand what it was that we actually did. Commonly when operating, one would realise that a small crowd had gathered behind to observe (Figure 2). Whilst we were unable to formally assess improved efficiency, it was observed that as the scrub team could better see and understand the procedure for which they were assisting, they provided an enhanced service as they better anticipated which instrument would be needed next, for example. Given that the aim of humanitarian camps such as the British Nepal Otology Service is to demonstrate technique and develop self-sufficiency of the local team, the endoscopic approach appeared to serve this goal well.

Arguably, the provision of a video monitor screen for attachment to the operating microscopes would achieve a similar outcome. It would not, however, be possible with the microscopes at our disposal at the British Nepal Otology Service, and would still be affected by the issues discussed previously. The one shared view, as obtained with the endoscope and camera setup, allows the surgeon, scrub team and as many observers as present to all observe the procedure on an equal footing.

In the out-patient department, we were able to review the cases for the next day. By displaying the image of potential cases on the screen, all could partake in the discussion (Figure 3). This again improved our ability to teach. It of course also proved a source of fascination to the patients themselves.

The future potential in this setting relates to telemedicine. The Storz system allows the recording of images onto a 


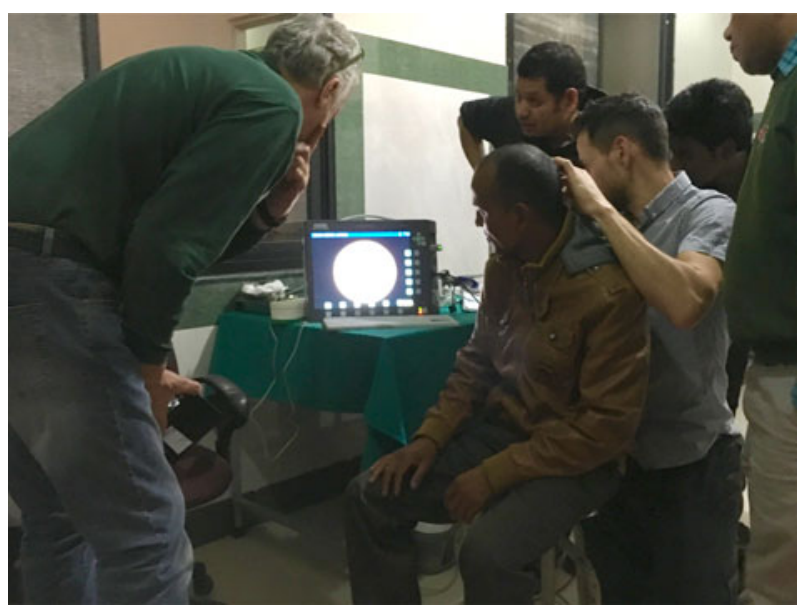

FIG. 3

The endoscope in clinic.

standard Universal Serial Bus (USB) stick. Outside of the camps, such images can then be emailed back to the UK for discussion about appropriate management.

\section{Discussion}

Formal studies are required to assess success rates and outcomes, to confirm that they are comparable to those of a microscopic approach. Comparison of operating time would also be interesting. This article was written to raise awareness and encourage forward thinking, rather than to prove the efficiency and success of the technique. However, it should be noted that this was not a form of experimentation. It is the author's usual practice in the UK to perform myringoplasties and limited cholesteatoma surgery entirely by an endoscopic approach.
In conclusion, endoscopic ear surgery in the ear camp setting might prove to be the way forward. The equipment, which is easily portable, provides improved visualisation of challenging tympanic membrane perforations. In addition, it allows for vast improvement in being able to teach, demonstrate and explain pathology and surgical techniques. Furthermore, it has the potential to establish regular telemedicine discussion of cases outside of the teams visiting ear camps.

\section{Acknowledgement}

The author, on behalf of the British Nepal Otology Service, would like to thank Dr Sybill Storz for her very generous donation of the equipment used.

\section{References}

1 Yong M, Mijovic T, Lea J. Endoscopic ear surgery in Canada: a cross-sectional study. J Otolaryngol Head Neck Surg 2016;45:4

2 Preyer S. Endoscopic ear surgery - a complement to microscopic ear surgery. HNO 2017;65(suppl 1):29-34

3 Choi N, Noh Y, Park W, Lee JJ, Yook S, Chung WH et al. Comparison of endoscopic tympanoplasty to microscopic tympanoplasty. Clin Exp Otorhinolaryngol 2017;10:44-9

Address for correspondence:

Mr Matthew P A Clark,

ENT Department

Wotton Lodge,

Gloucestershire Royal Hospital,

Great Western Road,

Gloucester GL1 3NN, UK

E-mail: Matthew.Clark@glos.nhs.uk

Mr M P A Clark takes responsibility for the integrity of the content of the paper

Competing interests: None declared 